\title{
Synthesis and photocatalytic activity of $\mathrm{ZnO} / \mathrm{ZnO}_{2}$ composite
}

\author{
Chin-Cheng Hsu, N.L. Wu* \\ Department of Chemical Engineering, National Taiwan University, Taipei 106, Taiwan, ROC
}

Received 20 July 2004; received in revised form 24 November 2004; accepted 15 December 2004

Available online 15 January 2005

\begin{abstract}
$\mathrm{ZnO} / \mathrm{ZnO}_{2}$ composite photocatalysts were synthesized by hydrothermal treatment at $120-180{ }^{\circ} \mathrm{C}$ of $\mathrm{ZnO}_{2}$, which in turn was obtained from an aqueous solution of $\mathrm{ZnSO}_{4}$ and $\mathrm{H}_{2} \mathrm{O}_{2}$. The composite particles showed morphology of $\mathrm{ZnO}$ prismatic crystallites with small $\mathrm{ZnO} 2$ granules "fused" at surface. Photocatalytic activity was characterized based on photocatalytic degradation of methyl orange under UV-light (300 nm) illumination, and the maximum activity was observed for the powder with an intermediate $\mathrm{ZnO}_{2}$-to- $\mathrm{ZnO}$ conversion. The enhanced activity, as compared with the pure $\mathrm{ZnO}$ crystallites, has been attributed to the presence of the intimately bonded $\mathrm{ZnO} / \mathrm{ZnO}_{2}$ surface hetero-structure. This study also points out a new approach to synthesize a composite photocatalyst containing strongly coupled constituents by phase transformation reactions among the constituents.
\end{abstract}

(C) 2004 Elsevier B.V. All rights reserved.

Keywords: Photocatalysis; $\mathrm{ZnO} ; \mathrm{ZnO}_{2}$; Hydrothermal synthesis

\section{Introduction}

$\mathrm{ZnO}$ is a wide-bandgap semiconductor having many applications, such as being a photocatalyst, transducer, varistor, phosphor, and transparent conductor [1], and wide varieties of synthesis techniques have thus been developed to produce this material in different forms [2]. They are, for instance, sol-gel process [3,4], hydrothermal process [5-7], chemical vapor deposition [8], and solution deposition [9,10]. It was particularly noticed that, in some of these processes $[3,9,10]$, $\mathrm{ZnO}$ was obtained by phase transformation reaction from a pre-existing $\mathrm{ZnO}_{2}$ matrix. Composites containing different proportions of these two phases can easily be obtained by varying the extent of the transformation. $\mathrm{ZnO}_{2}$ is also a semiconductor with a bandgap greater than that of $\mathrm{ZnO}$ [9]. Nevertheless, the photocatalytic property of such a composite system has not been investigated.

\footnotetext{
* Corresponding author. Tel.: +886 223627158; fax: +886223623040.

E-mail address: nlw001@ntu.edu.tw (N.L.Wu).
}

Indeed, combination of more than one semiconductors has in some cases resulted in composite photocatalysts that are superior to any of its constituents in terms of photocatalytic activity [11-16], and it is in general believed that the properties at the interfacial regions between the constituents play a crucial role $[11,12]$. One key factor to such a synergic effect therefore, resides in intimate contact between the different phases. Conventionally, this was carried out either by calcining mixtures of the precursors of the constituent elements $[13,14]$ or by depositing a second constituent phase onto the surface of the first, major one [15,16].

This work is thus motivated to explore the opportunity for enhanced photocatalytic activity in the $\mathrm{ZnO} / \mathrm{ZnO}_{2}$ system as compared with pure $\mathrm{ZnO}$. In brief, $\mathrm{ZnO}_{2}$ powder was first synthesized via a precipitation method and then subjected to hydrothermal treatment to form $\mathrm{ZnO}$. Composites contain different $\mathrm{ZnO} / \mathrm{ZnO}_{2}$ proportions were obtained by hydrothermal treatments of $\mathrm{ZnO}_{2}$ within the temperature range between 120 and $180^{\circ} \mathrm{C}$. Photocatalytic activity of the powders was characterized based on degradation of methyl orange [17]. The maximum activity was observed with an intermediate 
$\mathrm{ZnO} / \mathrm{ZnO}_{2}$ composition, which is associated with an intimately bonded $\mathrm{ZnO} / \mathrm{ZnO}_{2}$ surface hetero-structure.

\section{Experimental}

To synthesize $\mathrm{ZnO}_{2}$ powder, $500 \mathrm{ml}$ of $0.1 \mathrm{M}$ $\mathrm{ZnSO}_{4} \cdot 7 \mathrm{H}_{2} \mathrm{O}$ aqueous solution and $500 \mathrm{ml}$ of $0.1 \mathrm{M}$ $\mathrm{NH}_{4} \mathrm{OH}$ aqueous solution were mixed to obtain a white zinc oxy-hydroxyl precipitate. After being washed with excessive de-ionized water, the precipitate was introduced into a $1 \mathrm{M}$ $\mathrm{H}_{2} \mathrm{O}_{2}$ aqueous solution. The dispersion solution was then heated at $75^{\circ} \mathrm{C}$ for $2 \mathrm{~h}$ to result in $\mathrm{ZnO}_{2}$ powder, which was subsequently washed with excessive de-ionized water and finally dried at $65^{\circ} \mathrm{C}$ in air. The $\mathrm{ZnO} / \mathrm{ZnO}_{2}$ composite powders were synthesized by subjecting the $\mathrm{ZnO}_{2}$ powder to hydrothermal treatment. In a typical hydrothermal run, the $\mathrm{ZnO}_{2}$ powder was dispersed in de-ionized water in a stainless-steel autoclave with Teflon lining, and then heated at the selected temperature, ranging between 120 and $180^{\circ} \mathrm{C}$, for $2 \mathrm{~h}$. After the hydrothermal treatment, the precipitate was collected, washed with de-ionized water and finally dried at $65^{\circ} \mathrm{C}$.

X-ray diffraction (XRD; Mac-Science/MXP3) was employed to determine crystalline phases and their (average) crystallite size based on the Scherrer equation. The conversion of the transformation reaction was determined based on the intensity ratio between the $\left(\begin{array}{lll}1 & 0 & 2\end{array}\right)$ reflection of $\mathrm{ZnO}$ and (220) reflection of $\mathrm{ZnO}_{2}$. These two reflections, although not the strongest reflection for either of the compounds, were chosen because they do not overlap with other peaks. For this purpose, a correlation curve of the XRD intensity ratio versus powder composition was constructed using mixtures of single-phased $\mathrm{ZnO}$ and $\mathrm{ZnO}_{2}$ powders prepared via the same processes as described above with known compositions. The powder morphology was analyzed by scanning electron microscope (Hitachi S-800). Diffuse reflectance ultraviolet-visible (UV-vis) spectroscopic analysis was conducted on a Hitachi U3410 spectrometer, which is equipped with an integration sphere and $\mathrm{PbS}$ and photomultiplier detectors for photon detection. Deuterium and tungsten iodide lamps are used as the light sources for wavelength ranges of $185-340$ and $340-2500 \mathrm{~nm}$, respectively. The spectra were acquired at room temperature with $\mathrm{BaSO}_{4}$ as the reference.

Kinetic experiments were carried out by using a vertical tubular batch reactor made of quartz. During a typical run, the entire reactor was enclosed inside a UV-light house (Rayonet photochemical reactor, RPR-100), and the reactor was partially filled with an aqueous methyl orange solution, in which the oxide particles, in an amount of $0.25 \mathrm{~g} / 1$, were constantly dispersed by a magnetic stirrer. The light house was equipped with eight UV-light tubes (maximum intensity at $300 \mathrm{~nm}$, Fig. 1), each having a power of $12 \mathrm{~W}$. The powder was dispersed in the solution for $60 \mathrm{~min}$ in dark, in order to reach the adsorption equilibrium of methyl orange on the

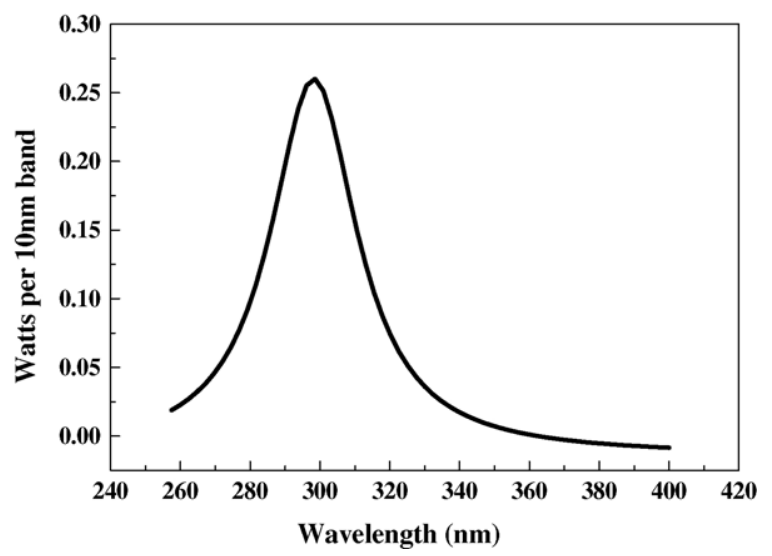

Fig. 1. Energy distribution of the UV-light source employed in the kinetic study.

powder, before UV-light was turned on. Small amounts of solution were retreated at the very beginning as well as intermittently during the course of reaction. The methyl orange concentration was determined by measuring the absorption at $\lambda=465 \mathrm{~nm}$, on a UV-vis spectroscopy (GENESYS ${ }^{\mathrm{TM}} 10$ SERIES). A correlation curve between methyl orange concentration and the absorption was pre-established.

\section{Results and discussion}

XRD showed that the powder produced by $\mathrm{H}_{2} \mathrm{O}_{2}$ at $75^{\circ} \mathrm{C}$ is single-phased $\mathrm{ZnO}_{2}$ having an Scherrer crystallite size of $5.4 \mathrm{~nm}$ (curve 1, Fig. 2). Upon hydrothermal treatment, the powder remained single-phased $\mathrm{ZnO}_{2}$ at $120^{\circ} \mathrm{C}$, while $\mathrm{ZnO}$ started to appear at $130^{\circ} \mathrm{C}$. With further increasing temperature, the amount of $\mathrm{ZnO}$ continued to grow at the expense of $\mathrm{ZnO}_{2}$ (Fig. 2). No any other phase was detected during the course of transformation, suggesting a simple one-step transformation mechanism:

$\mathrm{ZnO}_{2} \rightarrow \mathrm{ZnO}_{(\mathrm{s})}+\frac{1}{2} \mathrm{O}_{2(\mathrm{~g})}$

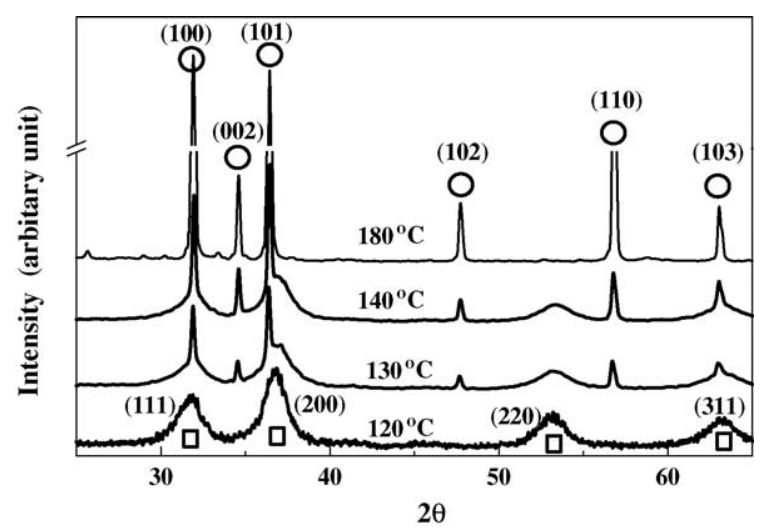

Fig. 2. XRD patterns of the powders synthesized at different temperatures. Key: ( $\bigcirc$ ) reflections of $\mathrm{ZnO}$; $(\square)$ reflections of $\mathrm{ZnO}_{2}$. 


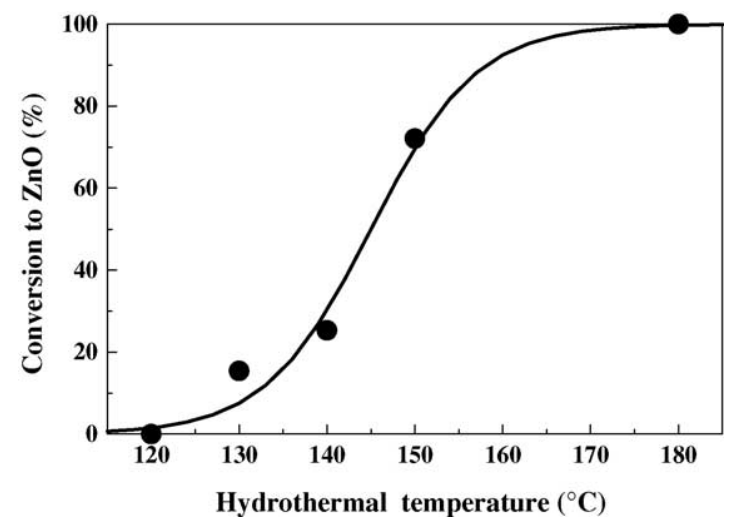

Fig. 3. Phase transformation conversion from $\mathrm{ZnO}_{2}$ to $\mathrm{ZnO}$ vs. hydrothermal temperature.

The conversion data (Fig. 3) indicate that the transformation accelerates at temperatures exceeding $140^{\circ} \mathrm{C}$, and the powder became single-phased $\mathrm{ZnO}$ after treatment at $180^{\circ} \mathrm{C}$. It was also noticed that the reflections of $\mathrm{ZnO}$ are very sharp, indicating large crystallites, even for the powders of low conversion, such as the 130 and $140^{\circ} \mathrm{C}$ samples. Calculation based on the Scherrer equation indicated average $\mathrm{ZnO}$ crystallite sizes exceeding $40 \mathrm{~nm}$ in all samples (exact values cannot be determined with high confidence because large error can easily arise from choosing the baseline of the XRD spectra due to the sharpness of the peaks).

SEM analysis showed that the $\mathrm{ZnO}_{2}$ starting powder has granular morphology with a rather uniform particle size of $\sim 0.25 \mu \mathrm{m}$ (Fig. 4a). On the other hand, the fully transformed, $180^{\circ} \mathrm{C}$ sample showed rod-like $\mathrm{ZnO}$ prisms having a length from $\sim 3$ to $5 \mu \mathrm{m}$ and $\sim 1 \mu \mathrm{m}$ in thickness (Fig. $4 \mathrm{~b}$ ). The $140{ }^{\circ} \mathrm{C}$ powder, which has a conversion of $25 \%$, shows a small number of large $\mathrm{ZnO}$ prisms (Fig. 4c) having a dimension of a few microns instead of large amount of small $\mathrm{ZnO}$ crystallites. This is consistent with the XRD results, which show very sharp $\mathrm{ZnO}$ peaks even at low conversion. These results may suggest that nucleation be the rate-limiting step for the transformation, and therefore, stable nuclei grow rapidly while the number of nuclei remains small. In addition, it was noticed that the surfaces of the $\mathrm{ZnO}$ crystallites in the partially transformed powders were mostly "fused" with $\mathrm{ZnO}_{2}$ granules (Fig. 4c). The surface density of the granules is abundant for the $140^{\circ} \mathrm{C}$ crystallites, but significantly decreases
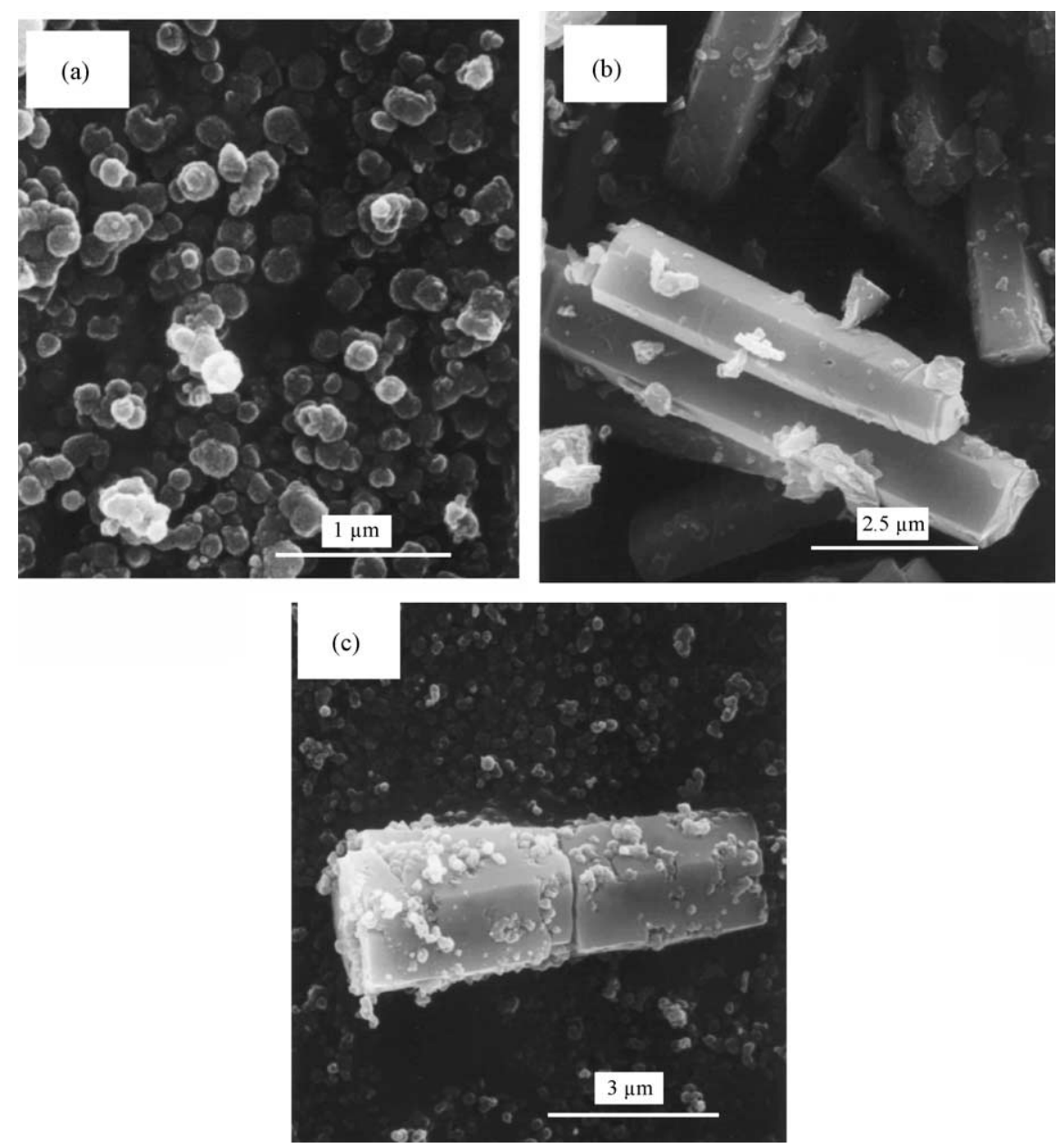

Fig. 4. SEM micrographs: (a) $\mathrm{ZnO}_{2}$; (b) $\mathrm{ZnO}$ obtained at $180^{\circ} \mathrm{C}$ and (c) $\mathrm{ZnO} / \mathrm{ZnO}_{2}$ at $140{ }^{\circ} \mathrm{C}$. 
as the transformation approaches completion at $180^{\circ} \mathrm{C}$ (Fig. 4b).

This is the first time $\mathrm{ZnO}_{2}$-to- $\mathrm{ZnO}$ transformation has been carried out under hydrothermal condition. Uekawa et al. [4] prepared $\mathrm{ZnO}_{2}$ by using the same procedures as in the present study but carried out the transformation reaction by firing $\mathrm{ZnO}_{2}$ under dry condition (the atmosphere was not explicitly specified but most likely was either air or oxygen). The resulted powders were $\mathrm{ZnO}$ nanoparticles of 19 and $41 \mathrm{~nm}$ after firing at 300 and $500{ }^{\circ} \mathrm{C}$, respectively. Heat treatment of highly crystallized $\mathrm{ZnO}_{2}$ thin film in air or vacuum $[9,10]$ also resulted in nanocrystalline $\mathrm{ZnO}$. These results are in great contrast with the $\mu \mathrm{m}$-sized $\mathrm{ZnO}$ crystallites obtained here. The difference may indicate that the nucleation step is greatly accelerated and no longer be rate limiting under their synthesis conditions. Thus, the $\mathrm{ZnO}$ crystallites containing surface $\mathrm{ZnO}_{2}$-granules are unique to the hydrothermally effected $\mathrm{ZnO}_{2}$-to- $\mathrm{ZnO}$ transformation. Direct hydrothermal synthesis of $\mathrm{ZnO}$ from $\mathrm{Zn}$ oxy-hydroxide precipitate does not create similar morphology [5-7].

The UV-vis spectra have been expressed in KubelkaMunk unit $(K / S)$, as shown in Fig. 5, where $K$ is the absorption coefficient, and $S$, the scattering coefficient. The starting $\mathrm{ZnO}_{2}$ powder showed an absorption edge at $300 \mathrm{~nm}$, corresponding to a bandgap of $4.12 \mathrm{eV}$. The bandgap is smaller, by nearly $0.1 \mathrm{~V}$, than that reported for a highly crystallized $\mathrm{ZnO}_{2}$ thin film prepared by a solution deposition method [10], and the difference may be because the $\mathrm{ZnO}_{2}$ powder synthesized here has poorer crystallinity. This is also indicated by the broad transition in absorption near the edge energy. When $\mathrm{ZnO}$ started to appear after hydrothermal treatment at $130^{\circ} \mathrm{C}$, the spectrum showed a two-step profile with an additional absorption at $395 \mathrm{~nm}$. With increasing hydrothermal temperature, the $\mathrm{ZnO}_{2}$-absorption decreased, while the $\mathrm{ZnO}$ absorption increased and shifted gradually to higher wavelengths. The profile finally showed single-step $\mathrm{ZnO}$ absorption with the absorption edge at $395 \mathrm{~nm}(3.13 \mathrm{eV})$ for the $180^{\circ} \mathrm{C}$ sample. These results are consistent with the XRD data in terms of the phase composition.

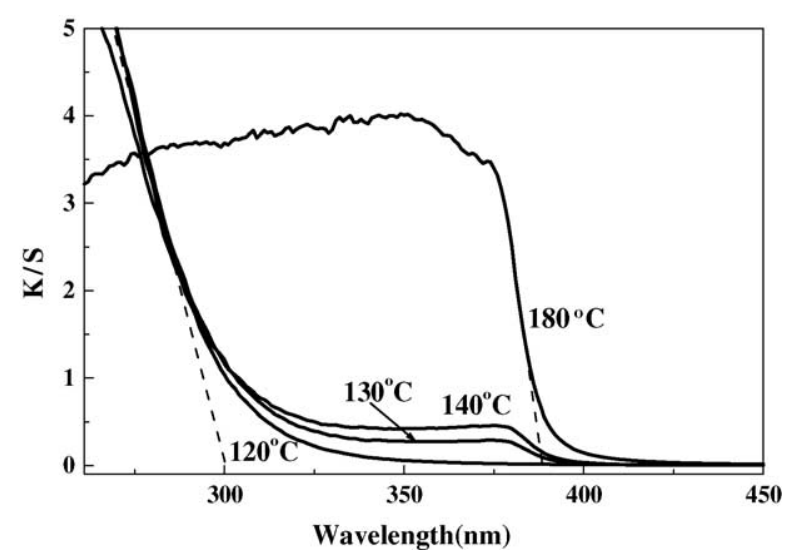

Fig. 5. UV-vis spectra of the powders synthesized at different temperatures.

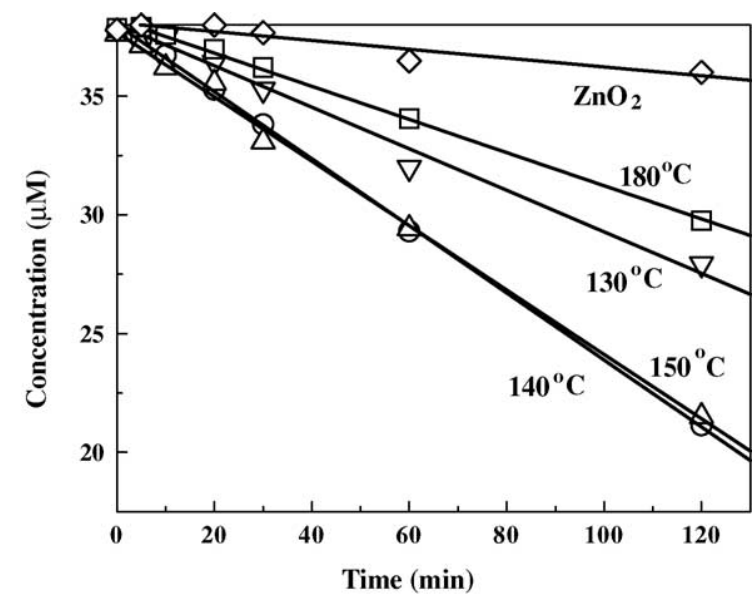

Fig. 6. Concentration of methyl orange vs. time under irradiation of UVlight.

The kinetic study was carried out by monitoring the absorption of the solution at $465 \mathrm{~nm}$ wavelength. A decrease in this absorption corresponds to degradation of polyaromatic rings in methyl orange to create mono substituted aromatics [17]. Fig. 6 shows methyl orange concentration versus time under irradiation for different powders. The $\mathrm{ZnO}_{2}$ powder, either fresh prepared or hydrothermally treated at $120^{\circ} \mathrm{C}$, exhibited very low activity. This is rather expected, because the absorption edge wavelength $(300 \mathrm{~nm})$ of the $\mathrm{ZnO}_{2}$ powder is the same as the maximum-intensity wavelength of the light source, and the efficiency for generating electrons and holes is hence expected to be very low. This may additionally be compounded by the poor crystallinity of the powder, which shortens the lifetimes of the excitons. For powders that were synthesized at $130^{\circ} \mathrm{C}$ or above, the methyl orange concentration was found to decrease almost linearly with time. The linearity suggests the zero-order reaction kinetics, and the slope is equal to the rate constant. The rate constant (Fig. 7) was found to first increase and then decrease with increasing $\mathrm{ZnO}$ content, showing a maximum for the powder synthesized within $140-150^{\circ} \mathrm{C}$. The presence of an optimum activity at an intermediate $\mathrm{ZnO} / \mathrm{ZnO}_{2}$ ratio may

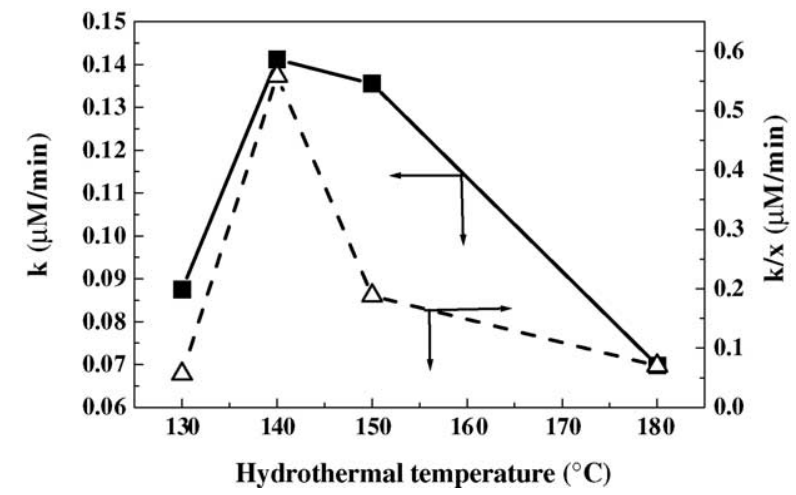

Fig. 7. Rate constant $(k)$ and normalized rate constant $(k / x)$ vs. hydrothermal temperature, where $x$ is the $\mathrm{ZnO}_{2}$-to- $\mathrm{ZnO}$ conversion, for photocatalyzed degradation of methyl orange. 
indicate an important role played by the interface of the two phases.

The physical and chemical mechanisms involved in semiconductor photocatalysis have been reviewed in detail in the literature (e.g., [11,12]). In brief, the processes involved can roughly be divided into three groups. The first group includes the physical processes taking place within the solid catalyst to result in a supply of charge carriers to the catalyst surface. They are photo-excited generation of electron in conduction band and hole in valence band, and diffusion and recombination of the generated charge carriers. The second group includes a series of surface reaction steps leading to chargetransfer at the solid-fluid interface. And the third group includes the transport processes of the chemical species (reactants/products) on the fluid side of the interface. As these three groups operate in series, the overall reaction kinetics is limited by the group that is the slowest, i.e., rate-limiting.

In the case where either the interfacial reaction or transport of chemical species on the fluid side is rate-limiting, the overall reaction rate would show non-zero reaction order. On the other hand, if the reaction rate is limited by the supply of the charge carriers on the solid side to the interface, one expects the reaction rate to exhibit zero-order concentration dependence. This is the case observed for the methyl orange degradation in the present study (Fig. 6). That is, for all the samples shown here, the overall reaction activity toward methyl orange degradation is determined by the total arriving rates of charge carriers at the catalyst surface.

As shown in Figs. 6 and 7, the photocatalytic activity does not show monotonic correlation with the hydrothermal temperature, i.e., with the amount of $\mathrm{ZnO}$. Rather, some of the partially transformed powders, particularly the 140 and $150^{\circ} \mathrm{C}$ powders, showed higher activities than the singlephased $\mathrm{ZnO}$ powder synthesized at $180^{\circ} \mathrm{C}$. To understand these differences, two factors are considered, including the total active surface area and the arrival flux (per unit time and area) of charge carriers to the catalyst surface. Because of the wavelength of the light source employed, we may consider that only the surface area of $\mathrm{ZnO}$ is of primary importance. As described early, the transformation reaction appeared to be nucleation-limited, and XRD and SEM results showed that the $\mathrm{ZnO}$ phase in different powders possess similar grain and particle sizes. As a result, the specific surface area of the $\mathrm{ZnO}$ phase should be similar in all powders, and therefore, the total active surface area should increase with increasing amount of $\mathrm{ZnO}$. Thus, the non-monotonic trend in catalytic activity is not likely due to the variation in active surface area.

Assuming similar specific surface areas of $\mathrm{ZnO}$ in these powders, the value of the reaction constant divided by transformation conversion (right $y$-axis, Fig. 7), referred as the normalized rate constant, then measures the specific surface activity, and in this case the arrival flux of charge carriers to the surface. The first rise in specific surface activity observed for hydrothermal temperature between 130 and $140{ }^{\circ} \mathrm{C}$ may be due to improved crystallinity of the synthesized $\mathrm{ZnO}$ phase with an increase in hydrothermal temperature. On the other hand, at higher hydrothermal temperatures, the specific surface activity are in the order of $140>150>180^{\circ} \mathrm{C}$. This trend was found to be correlated with the amount of the $\mathrm{ZnO}_{2}$ granules present on the $\mathrm{ZnO}$ crystallites. That is, a higher specific surface activity corresponds to a greater amount of $\mathrm{ZnO}_{2}$ surface granules. It is suggested that the $\mathrm{ZnO} / \mathrm{ZnO}_{2}$ interface may play an important role in enhancing the arrival flux of charge carrier to the $\mathrm{ZnO}$ surface. One possible effect due to the presence of the $\mathrm{ZnO}_{2}$ granules is that the difference in band structure between $\mathrm{ZnO}$ and $\mathrm{ZnO}_{2}$ could result in potential gradient at the interface, which facilitates separation and hence reduces recombination between the excited holes and electrons. In addition, the structural defects created near the $\mathrm{ZnO} / \mathrm{ZnO}_{2}$ interfaces could also serve as favorable trap sites of the excitons to reduce their recombination. In either case, a higher flux of charge carrier resulted from a longer carrier life time. Detailed photo-absorption and cathodoluminescence spectroscopic analyses on individual $\mathrm{ZnO}$ crystallites are underway.

\section{Conclusion}

$\mathrm{ZnO} / \mathrm{ZnO}_{2}$ composite photocatalyst containing $\mathrm{ZnO}$ crystallites bonded with surface $\mathrm{ZnO}_{2}$ granules have been synthesized by a hydrothermal treatment of $\mathrm{ZnO}_{2}$. The composite has shown a superior photocatalytic activity as compared with either of its constituents toward degradation of methyl orange. The enhancement has been ascribed to the presence of either structural or energetic heterogeneity within the interfacial regions between the two oxides. This study has also pointed out a new approach to synthesize a composite photocatalyst containing strongly coupled constituents, namely via one or multiple steps of transformation reaction among the constituents.

\section{Acknowledgment}

The work is supported by the Ministry of Economic Affair under grant 93-EC-17-A-09-S1-019.

\section{References}

[1] D.C. Look, Recent advances in $\mathrm{ZnO}$ materials and devices, Mater. Sci. Eng. B80 (2001) 383-387.

[2] Z.L. Wang, Nanostructures of zinc oxide, Mater. Today (2004) 26-33.

[3] M.S. Tokumoto, S.H. Pulcinelli, C.V. Santilli, V. Briois, Catalysis and temperature dependence on the formation of $\mathrm{ZnO}$ nanoparticles and of zinc acetate derivatives prepared by the sol-gel route, J. Phys. Chem. B 107 (2003) 568-574.

[4] N. Uekawa, J. Kajiwara, N. Mochizuki, K. Kakegawa, Y. Sasaki, Synthesis of $\mathrm{ZnO}$ nanoparticles by decomposition of zinc peroxide, Chem. Lett. (2001) 606-607.

[5] H. Xu, H. Wang, Y.C. Zhang, S. Wang, M.K. Zhu, H. Yan, Asymmetric twinning crystals of zinc oxide formed in a hydrothermal process, Cryst. Res. Technol. 38 (2003) 429-432. 
274

C.-C. Hsu, N.L. Wu / Journal of Photochemistry and Photobiology A: Chemistry 172 (2005) 269-274

[6] B. Liu, H.C. Zeng, Hydrothermal synthesis of $\mathrm{ZnO}$ nanorods in the diameter regime of $50 \mathrm{~nm}$, J. Am. Chem. Soc. 125 (2002) 4430-4431.

[7] S. Music, S. Popovic, M. Maljkovic, D. Dragcevic, Influence of synthesis procedure of the formation and properties of zinc oxide, J. Alloy Comp. 347 (2002) 324-332.

[8] M.F. Ogawa, Y. Natsume, T. Hirayama, H. Sakata, Preparation and electrical properties of undoped zinc-oxide films by CVD, J. Mater. Sci. Lett. 9 (1990) 1351-1353.

[9] S. Lindross, M. Leskela, Growth of zinc peroxide $\left(\mathrm{ZnO}_{2}\right)$ and zinc oxide $(\mathrm{ZnO})$ thin films by the successive ionic layer adsorption and reaction-SILAR-technique, Inter. J. Inorg. Mater. 2 (2000) 197-201.

[10] M. Ortega-Lopez, A. Avila-Garcia, M.L. Albor-Aguilera, V.M.S. Resendiz, Improved efficiency of the chemical bath deposition method during growth of $\mathrm{ZnO}$ thin films, Mater. Res. Bull. 38 (2003) 1241-1248.

[11] A.L. Linsebigler, G. Lu, J.T. Yates Jr., Photocatalysis on $\mathrm{TiO}_{2}$ surfaces: principles, mechanisms, and selected results, Chem. Rev. 95 (1995) 735-758.
[12] A.J. Nozik, R. Memming, Physical chemistry of semiconductor/liquid interfaces, J. Phys. Chem. 100 (1996) 13061-13078.

[13] M. Zhang, T. An, X. Hu, C. Wang, G. Sheng, J. Fu, Preparation and photocatalytic properties of a nanometer $\mathrm{ZnO}-\mathrm{SnO}_{2}$ coupled oxide, Appl. Catal. A 260 (2004) 215-222.

[14] T. Tani, L. Madler, S.E. Pratsinis, Synthesis of $\alpha$-Willemite nanoparticles by post-calcination of flame-made zinc oxide/silica composites, Part. Syst. Charact. 19 (2002) 354-358.

[15] K.R. Gopidas, M. Bohorquez, P.V. Kamat, Photophysical and photochemical aspects of coupled semiconductors: charge-transfer processes in colloidal cadmium sulfide-titania and cadmium sulfidesilver(I) iodide systems, J. Phys. Chem. 94 (1990) 64356440.

[16] I.H. Tseng, J.C.S. Wu, H.Y. Chou, Effects of sol-gel procedures on the photocatalysis of $\mathrm{Cu} / \mathrm{TiO}_{2}$ in $\mathrm{CO}_{2}$ photoreduction, J. Catal. 221 (2004) 432-440.

[17] S. Al-Qaradawi, R. Salman, Photocatalytic degradation of methyl orange as a model compound, J. Photochem. Photobio. A: Chem. 148 (2002) 161-168. 